\title{
An Approximate Queueing Model for Limited-Range Wavelength Conversion in an OBS Switch
}

\author{
Vishwas S Puttasubbappa and Harry G Perros \\ North Carolina State University, \\ Computer Science Department, Raleigh NC 27695-7534 \\ \{vsputtas, hp\}@csc.ncsu.edu
}

\begin{abstract}
We develop an analytical model for modeling limited-range wavelength conversion employed in an OBS switch. The system is modeled as a simultaneous resource possession problem. We propose a productform solution which calculates approximate blocking probabilities for degree of conversion $d=1,2$ and for large number of wavelengths. We then propose an approximate model for large values of $d$. The output of our model was verified with simulation results.
\end{abstract}

\section{Introduction}

Optical Burst Switching (OBS) is one of the promising technologies to realize the next generation all-optical Internet [3] $8,[15$. In OBS, the contention issue that arises at the switches due to simultaneous arrival of several control packets can be minimized by using wavelength conversion, Fiber Delay Line (FDL) buffers and deflection routing. A combination of these three techniques can also be employed to reduce blocking of data bursts. C. Gauger [5] examined the performance of OBS nodes which employ wavelength converter pools and FDL buffers for contention resolution. Several strategies based on different ordering of probing converter pools and FDL buffers have been proposed to optimize performance such as minimizing delay or the number of converters.

In this paper, we focus on wavelength conversion to reduce contention among data bursts. We consider only the case of limited-range wavelength conversion. In limited-range wavelength conversion, data bursts arriving on a wavelength can be converted to a fixed set of wavelengths above and below the original wavelength. The degree of conversion $d$ defines the number of target wavelengths for conversion on either side of the original wavelength. Thus an incoming data burst can be converted to a total of $(2 * d+1)$ destination wavelengths. Previous studies on limited-range wavelength conversion for lightpaths include [4, [12] and [14 for an OBS network. Recently, Akar and Karasan 2] proposed a method to exactly calculate the blocking probabilities in an OBS switch with partial wavelength conversion, i.e number of converters available being less than the number of wavelengths. 
Previous studies in OBS which dealt with limited-range wavelength conversion focused on computing link blocking probabilities and path blocking probabilities [14. They also assumed Poisson arrivals for their model. In this paper, we focus on a single OBS switch. We assume sources to be of ON-OFF nature.

We develop an analytical model to determine the blocking probabilities for data bursts in a core OBS switch which employs limited-range wavelength conversion to resolve contention among data bursts. We assume the absence of FDLs and deflection routing. The blocking probabilities obtained from this queueing model are approximate. We propose a product-form solution from which blocking probabilities can be computed for large number of wavelengths but only for $d=1,2$. We then develop a large scale approximation technique which can be applied to large values of $w$ and $d$.

The paper is organized as follows: Section 2 describes the architecture of the core OBS switch that we have assumed for our model. In Section 3, we present an approximate queueing model for limited-range wavelength conversion for the case of small $d$. In Section 4, we describe an approximate model for large systems. In Section 5, we compare the outputs of our analytical models with the simulation results, and in Section 6, we draw some conclusions.

\section{The OBS Switch}

The core OBS switch is comprised of $k$ incoming and $k$ outgoing fibers, the switching fabric and wavelength converters. Such a switch can be implemented using several architectures [13 10, 11. Each output fiber has a set of wavelength converters $(c)$ that can be used by bursts traveling out of that particular fiber. The bandwidth in each fiber is partitioned into several wavelengths $(w)$ using Wavelength Division Multiplexing (WDM).

In this paper, we model one such output fiber with its own set of wavelength converters. Each outgoing wavelength $\lambda_{i}, i=1,2, \ldots, w$, has $k$ number of incoming wavelengths $\lambda_{i}$, one per input fiber, targeted into it. Part of the bursts arriving in each of the $k$ input wavelengths $\lambda_{i}$ is switched to the destination wavelength $\lambda_{i}$ of the output fiber under study. The remaining bursts are switched through other output fibers. An incoming burst on $\lambda_{i}$ will try to be scheduled on its home wavelength $\lambda_{i}$ in the outgoing fiber. In case the home wavelength is busy, the burst tries to occupy adjacent wavelengths in the range $d$, i.e $\lambda_{i} \pm d$, if a converter in the common converter pool per output fiber is available. The method of trying to occupy an alternate wavelength will be explained in the analytical model section.

\section{The Queueing Model for Small $d$}

The Markov process for limited-range wavelength conversion does not have a product-form solution [9]. In view of this, we propose an approximate solution for the problem of computing blocking probabilities in an OBS switch that employs 
limited-range wavelength conversion. The queueing problem is modeled as a simultaneous resource possession problem as follows:

Let us consider a three wavelength example to illustrate our method. The original model is decomposed into sub-systems as depicted schematically in figure 1 .
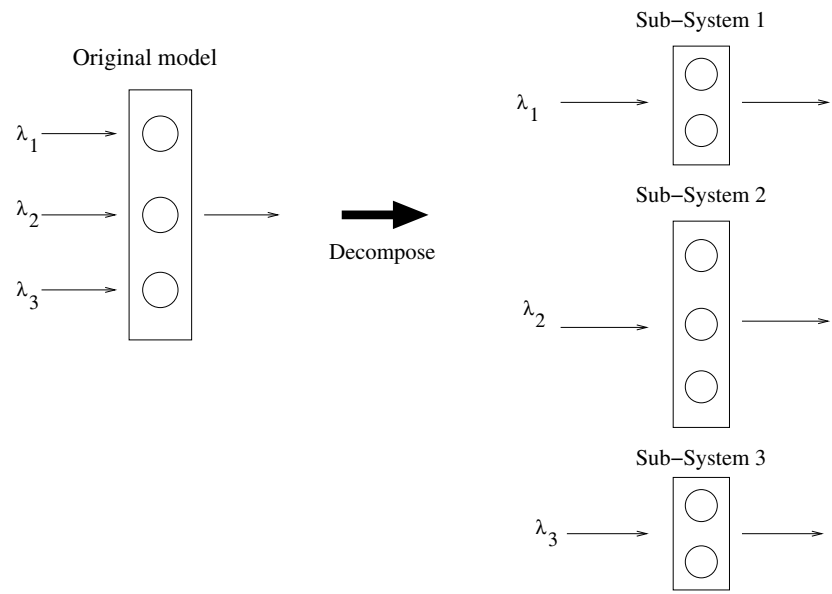

Fig. 1. A three node model

Figure 1 depicts the decomposition of a system with $w=3$ and $d=1$ into three sub-systems $\bar{n}_{1}, \bar{n}_{2}$ and $\bar{n}_{3}$ each being an Erlang loss queue. The number of sub-systems is equal to the number of wavelengths $w$. Each sub-system $\bar{n}_{i}$ represents a home wavelength $\lambda_{i}$ and neighboring wavelengths into which a burst coming on the home wavelength can be converted into. We represent each wavelength with a server in the Erlang loss queue sub-system. Thus, each sub-system has number of servers ranging from $(d+1)$ to $(2 * d+1)$. The border sub-systems $\bar{n}_{1}$ and $\bar{n}_{3}$ have two servers and the central sub-system $\bar{n}_{2}$ has three servers. The state of each server is represented by $n_{i, j} \epsilon\{0,1\}$, where $i$ is the sub-system and $j$ is the wavelength index. Thus, $\bar{n}_{1}=\left\{n_{1,1}, n_{1,2}\right\}, \bar{n}_{2}=\left\{n_{2,1}, n_{2,2}, n_{2,3}\right\}$ and $\bar{n}_{3}=\left\{n_{3,2}, n_{3,3}\right\}$.

The state of the system is given by the union of states of servers in all the sub-systems combined. Thus, for the three node example, the state of the system is represented by the tuple $\left(\bar{n}_{1}, \bar{n}_{2}, \bar{n}_{3}\right)=\left(n_{1,1}, n_{1,2}, n_{2,1}, n_{2,2}, n_{2,3}, n_{3,2}, n_{3,3}\right)$.

Let $p\left(\bar{n}_{1}, \bar{n}_{2}, \bar{n}_{3}\right)$ be the probability of the system being in one such state. Then, we have

$$
p\left(\bar{n}_{1}, \bar{n}_{2}, \bar{n}_{3}\right)=\frac{1}{G} * p\left(\bar{n}_{1}\right) * p\left(\bar{n}_{2}\right) * p\left(\bar{n}_{3}\right)
$$

or,

$$
\begin{gathered}
p\left(n_{1,1}, n_{1,2}, n_{2,1}, n_{2,2}, n_{2,3}, n_{3,2}, n_{3,3}\right)=\frac{1}{G} * p\left(n_{1,1}, n_{1,2}\right) \\
* p\left(n_{2,1}, n_{2,2}, n_{2,3}\right)(2) \\
* p\left(n_{3,2}, n_{3,3}\right)
\end{gathered}
$$

where $G=\sum p\left(\bar{n}_{1}\right) * p\left(\bar{n}_{2}\right) * p\left(\bar{n}_{3}\right)$ summed over all feasible states. 
The problem has been decomposed to a granularity which allows us to apply the rules of simultaneous resource possession.

Rule 1: A wavelength server can be occupied in only one sub-system Rule 2: The number of conversions cannot exceed $c$

Thus we have,

$$
\begin{gathered}
n_{1,1}+n_{2,1}=1 \\
n_{1,2}+n_{2,2}+n_{3,2}=1 \\
n_{2,3}+n_{3,3}=1 \\
n_{1,2}+n_{2,1}+n_{2,3}+n_{3,2} \leq c
\end{gathered}
$$

The wavelength occupancy constraint (Rule 1) and the converters constraint (Rule 2) reduce the state space from which the solution is computed. The computation of the individual sub-system probabilities $p\left(\bar{n}_{x}\right)$, where $x \epsilon\{1 \ldots w\}$ will be explained in the sub-section 3.2 .

When we generalize our model to any number of wavelengths $w$, we have,

$$
p\left(\bar{n}_{1}, \bar{n}_{2}, \bar{n}_{3}, \ldots, \bar{n}_{w}\right)=\frac{1}{G} * p\left(\bar{n}_{1}\right) * p\left(\bar{n}_{2}\right) * p\left(\bar{n}_{3}\right) * \ldots * p\left(\bar{n}_{w}\right)
$$

The model is further decomposed so as to include the constraints of wavelength occupancy and the number of available converters. Each subsystem thus becomes:

$$
\bar{n}_{i}=\left(n_{i, i-d}, n_{i, i-d+1}, \ldots, n_{i, i}, \ldots, n_{i, i+d-1}, n_{i, i+d}\right)
$$

and in $n_{i, j}, \mathrm{i}, \mathrm{j} \in\{1 \ldots w\}$

The constraints for simultaneous resource possession are:

$n_{i, i-d}+n_{i, i-d+1}+\ldots+n_{i, i-1}+n_{i, i}+n_{i, i+1}+\ldots+n_{i, i+d-1}+n_{i, i+d}=1 \forall i=1,2, \ldots, w$

$$
\sum_{i=1, j=i-d, j \geq 1}^{i=w, j=i+d} n_{i, j} \leq c
$$

The computation of $\mathrm{G}$ is a complicated task because of the state space explosion with large values of $w$ and $d$. We shall describe the approach we take for the computation of $\mathrm{G}$ in sub-section 3.3. Once the probability of the system existing in each state has been determined, the blocking probability of each wavelength is then the sum of all the corresponding blocking states.

\subsection{The Arrival Process}

We use the IDLE-ON arrival process shown in figure 2 to generate bursts on a single incoming wavelength $\lambda_{i}$. The IDLE and ON periods are exponentially distributed with a mean of $\frac{1}{\nu}$ and $\frac{1}{\mu}$ respectively. A single ON period generates a single burst. An IDLE period is followed by the ON period and vice-versa. 


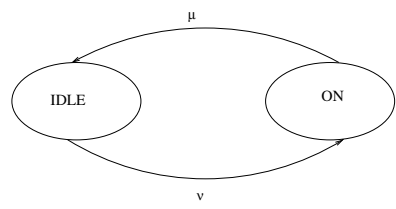

(a) Single IDLE-ON source

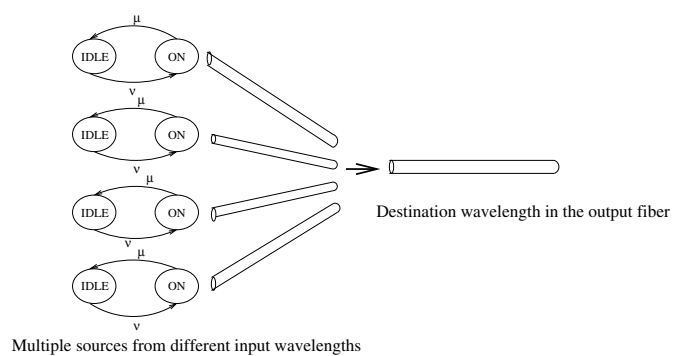

(b) Multiple IDLE-ON sources

If a burst is dropped, the source returns to the IDLE state. Since there are $k$ input fibers, the burst arrival process from all the $k$ incoming wavelengths $\lambda_{i}$ is the superposition of $k$ single IDLE-ON sources as shown in figure 2(b) We assume that all the $k$ arrival IDLE-ON sources are identical. We also ass ume in this paper that the superposition process is identical for all wavelengths $\lambda_{i}$, $i=1 \ldots w$.

As described in section 2, we are modeling a single output fiber. Each wavelength in the fiber will only have some of the traffic directed towards it from its $k$ corresponding input wavelengths. Consequently, the IDLE period is assumed to have been appropriately extended so that only the bursts destined to the outgoing wavelength in the fiber are modeled.

\subsection{Computing the Probabilities for Each Sub-system}

In each sub-system, a customer is allocated to the home server. In case it is busy, a free immediate adjacent server is chosen with probability 0.5 . If both are busy, the next set of adjacent servers are scanned for an empty server. The probabilities for each sub-system can be obtained from the birth-death process and lacks a closed-from expression [9]. On increasing the values of $w$ and $d$, it gets difficult to solve a Markov chain for each sub-system. On close examination, it can be seen that adding a few transitions to the birth-death can get us a closed-form expression, which is the Erlang system or the Engset system depending on the assumption regarding the arrival process. By using either of these systems, we can get away with the problem of marking the servers. Thus, we can assume that the servers are allocated randomly. This gives rise to a product-form solution which is an approximation to the probability terms $p\left(\bar{n}_{x}\right)$ in equation 7 .

In equation 2 , the probability terms on the right hand side are determined using the Engset model. If $n$ is the number of servers, $k$ the number of input fibers and $\mu, \nu$ are as described in the arrival process, the probability that there are $i$ customers in such a $M / M / n / n / k$ system is given by: 


$$
\pi_{i}^{*}=\frac{\left(\begin{array}{c}
k-1 \\
i
\end{array}\right)\left(\frac{\nu}{\mu}\right)^{i}}{\sum_{j=0}^{n}\left(\begin{array}{c}
k-1 \\
j
\end{array}\right)\left(\frac{\nu}{\mu}\right)^{j}}
$$

The Engset probabilities of equation 11 are those as seen by an arrival.

The approximated probabilities that a specific set of $i$ servers is occupied in a sub-system is given by:

$$
\pi_{i}=\frac{\pi_{i}^{*}}{\left(\begin{array}{c}
n \\
i
\end{array}\right)}
$$

The product of such terms if the state is valid can be added to compute $G$. The blocking probability of a particular wavelength is finally computed by summing up all the appropriate blocking states.

\subsection{Computation of $\mathrm{G}$}

We now describe the approach taken to compute the normalization constant G. By brute-force enumeration, it takes $\mathrm{O}\left(w^{w}\right)$ to cover the entire state space and determine the blocking probabilities for each wavelength. It is clear that brute-force enumeration cannot be used to compute $G$ for large values of $w$ and $d$. We have determined that this approach can be taken to solve for $d=1,2$ and very small values of $w$. For larger values of $d$, we propose the large scale approximation technique described in section 4.

\subsection{Large Number of Wavelengths}

In this section, we describe the method for extending the computation of blocking probabilities for large number of wavelengths where $d=1,2$.

As was described in section 3.3 , we can compute $G$ by brute-force enumeration only for very low values of $d$ and $w$. However, there are some properties of blocking probabilities with increasing number of wavelengths that we can make use of to compute blocking probabilities for these large systems.

Figure $3(\mathrm{a})$ plots the simulation results for the blocking probability of the middle wavelength of the system with increasing number of $w$ in the system. The number of converters increases proportionally with the number of wavelengths. It can be seen that with increase of $w$, there is a very small gradual decrease in the blocking probability. It can also be seen that the trend is similar for different arrival rates. We recall that we have assumed an identical superimposed arrival process to each wavelength.

Figure 3(b) plots the simulation results for the blocking probabilities for all the wavelengths in the system, for two different cases. We assume that each wavelength is fed by the same superposition arrival process. It can be seen that the border wavelengths have the highest blocking since the degree of conversion is smaller for them compared to the center wavelengths. In the following sections, we focus only the blocking probabilities of the middle wavelength and it can be noted that the border wavelengths have higher values. Also, we assume symmetric traffic wherein each wavelength is fed by the same arrival process resulting in the same arrival rate. 


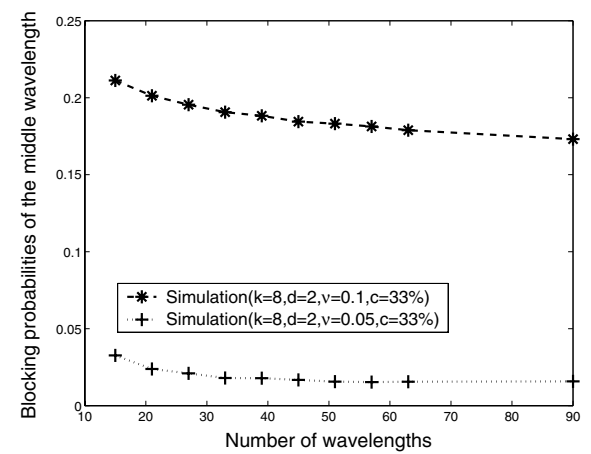

(a) proportional use of converters

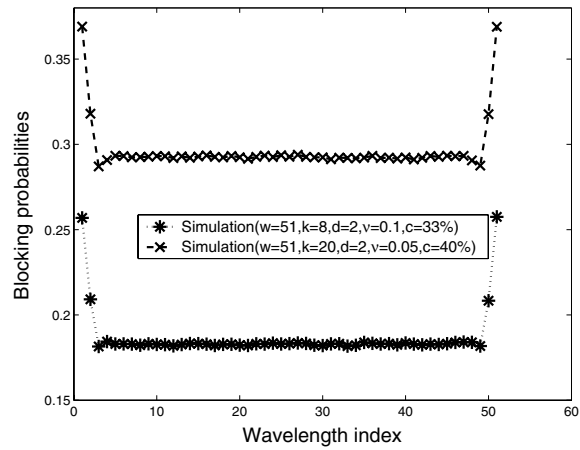

(b) Blocking probabilities for the complete spectrum

Fig. 3. Large number of wavelengths

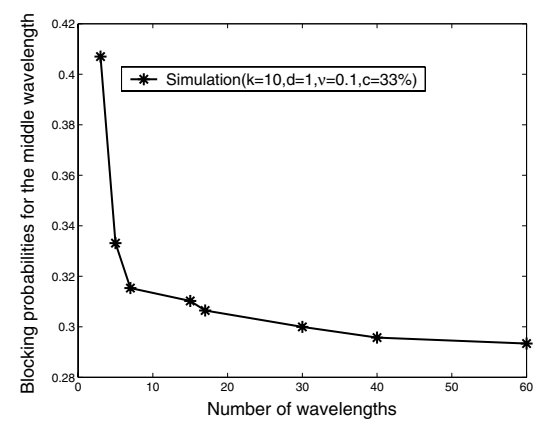

(a) Exponential decay

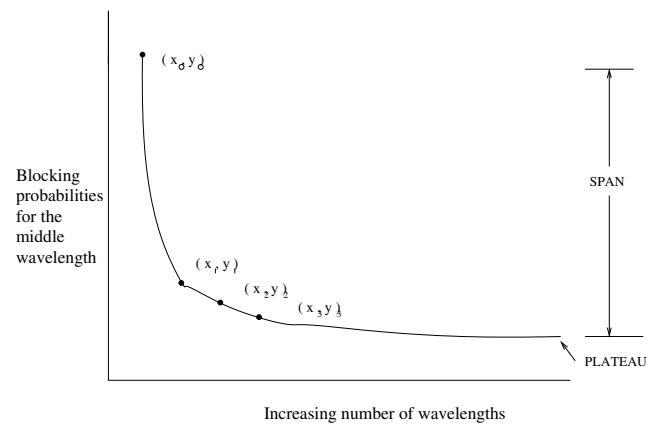

(b) Four-point method

Fig. 4. Large number of wavelengths

\subsection{The Four-Point Method}

From the previous section, it can be seen that a smaller system (smaller $w$ ) with a proportionally smaller number of converters can be made use of to get an approximate value of the blocking probabilities, which then slowly decreases with increasing $w$.

Figure 4(a) plots the simulation results for the blocking probability of the middle wavelength with increasing number of wavelengths as in figure $3(\mathrm{a})$, but starting with very few wavelengths. It can be seen that the distribution is very similar to the exponential distribution. In the four-point method, we use the brute-force enumeration described in section 3.3 to calculate the initial 4 points on the curve. We then use these points to determine the value of the plateau $P$ of the curve, see figure 4(b) 
Let $\left(x_{0}, y_{0}\right),\left(x_{1}, y_{1}\right),\left(x_{2}, y_{2}\right)$ and $\left(x_{3}, y_{3}\right)$ be the initial 4 points on the curve. The size of the system $W$ is $(2 * d+1),(2 * d+3),(2 * d+5)$ and $(2 * d+7)$ respectively. The converters are proportionally decreased from their initial value in the original system $w$. A one phase exponential decay function with the initial 4 points can be used to determine $P$, see [6], which is the blocking probability for large number of $w$ and for $d=1,2$.

\section{Large Scale Approximation}

The methods discussed in section 3 can only be applied for $d=1,2$. In this section, we describe an approximate model for large $d$. The schematics of the model are shown in figure 5.

The system is decomposed into two loss queues. The first one, referred to as the server bottleneck and the second one as the converter bottleneck. The server (i.e. wavelengths) bottleneck is used to determine the blocking due to lack of servers for a given $d$, and the converter bottleneck is used to determine blocking due to lack of converters $c$. The server bottleneck and the converter bottleneck are modeled separately and independence is assumed between them.

Figure 6] forms the basis for modeling the server bottleneck. The original system of size $w$ and $d$ with limited-range conversion has been transformed to a modified system of size $(2 * d+1)$ with full-range conversion. As was stated earlier in subsection 3.5 , the effect of border wavelengths tends to decrease as the system size considered, i.e the number of wavelengths $w$, increases. It was observed that this increase of system size can be approximately modeled by conversion from limited-range to full-range with $w=(2 * d+1)$. Further, the number of converters for the $(2 * d+1)$ system is proportionally reduced from the original system. A numerical example of this is shown in figure 7 where

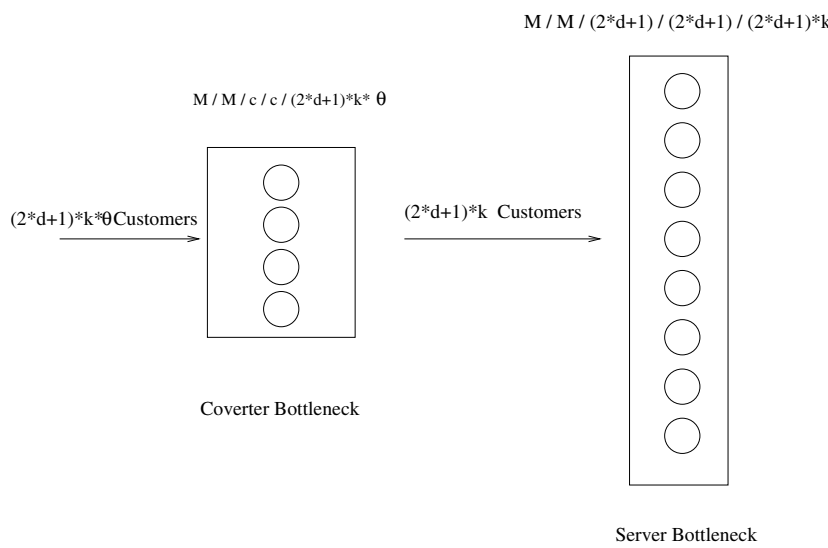

Fig. 5. Large scale approximation 


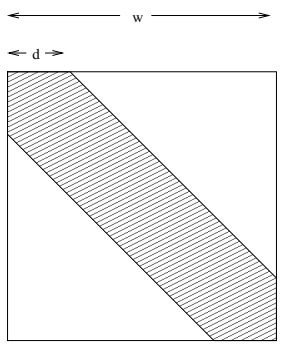

Original System (Limited-Range)

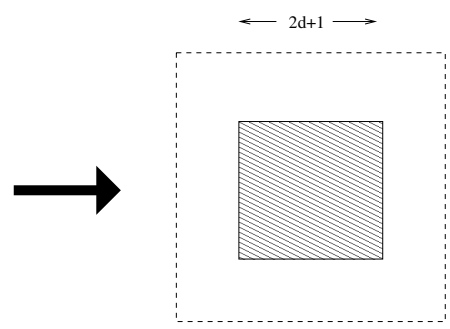

Modified System (Full-Range)

Fig. 6. Conversion from limited-range to full-range conversion

the $y$ axis denotes the average blocking probability of all the wavelengths. We use the same superposed arrival process to each wavelength as was described in section 3.1 .

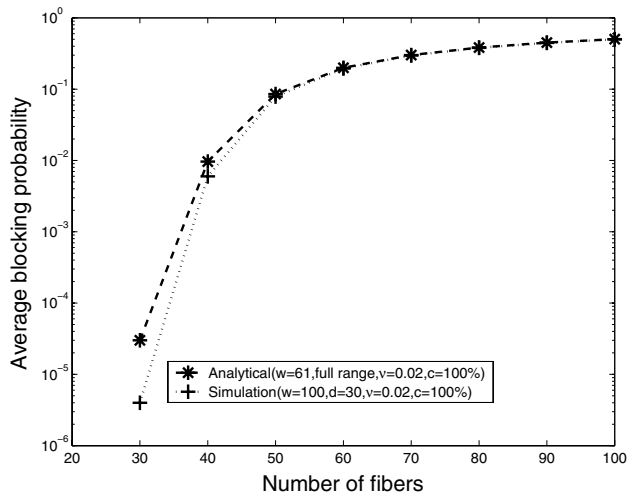

Fig. 7. Full-range conversion

The server bottleneck is modeled by an Engset system $M / M /(2 * d+1) /(2 *$ $d+1) /((2 * d+1) * k)$. The converter bottleneck is also modeled as an Engset system. But the percentage of $((2 * d+1) * k)$ customers, $\theta$, that have to use the converter pool needs to be determined. The approximate $\theta$ is determined as follows:

probability (an arrival using a converter)

$=$ probability (its destination wavelength is busy) $=p_{\text {Blocking }}(M / M / 1 / 1 / k)$

Therefore,

No. of arrivals that use a converter $=(2 * d+1) * k * p_{\text {Blocking }}(M / M / 1 / 1 / k)$

$$
=(2 * d+1) * k * \theta
$$


Thus, the Engset system for converter bottleneck becomes

$$
M / M / c / c /((2 * d+1) * k * \theta) .
$$

If $p_{B s}$ is the probability that a burst gets blocked at the servers and $p_{B c}$ is the probability that a burst gets blocked at the converter pool, then, because of the independence assumption the probability that a burst gets blocked is given by:

$$
p_{B}=1-\left(1-p_{B s}\right)\left(1-p_{B c}\right)
$$

\section{Results}

An event based simulation model was constructed to evaluate the results of the analytical model. The simulation results were plotted with $95 \%$ confidence interval estimated by the method of batch means, see Perros [7. Each batch is completed when every wavelength has 30,000 bursts arriving at it. The confidence intervals are very tight and are not discernible in the graphs. A software called GraphPad Prism 1] was used to fit a one phase exponential decay function with the initial 4 points as described in the four-point method.

The blocking probability of the center wavelength was calculated by varying $\nu$ (which varies the arrival rate), the number of fibers $k$, the degree of conversion, $d$ and the number of wavelengths, $w$. Both analytical results and simulation results have been plotted. We use the four-point method for $d=1,2$ and large scale approximation for larger $d$. Only a subset of these results are shown here. Please refer to [9] for the complete set.

The way we take the initial 4 points differs for $d=2$. To get the fourth point, we have to consider a system of size $(2 * d+7)=11$. By brute-force enumeration, it is not possible to explore all the states of such a system within reasonable time. So, we get the fourth point via simulation, which takes far lesser time. We note that the analytical values are much closer to the simulation ones than in the case of $d=1$. However, the range for very good accuracy seem to be between $10^{-1}$ and 1 . For lower blocking probabilities it is hard to fit a curve because of the gap between the three analytical points and the fourth simulation point.

Figure 8(f) plots the simulation results for large values of $d$. It can be seen that increasing $d$ is most effective at low arrival rates and/or at high \% conversion. Increasing $d$ does not have a big impact when blocking probabilities are high.

\section{Conclusion}

In this paper, we proposed an approximate solution for limited-range wavelength conversion in an OBS switch. The problem was modeled as a simultaneous resource possession problem and an approximate product-form solution was proposed. This solution could be applied for very small values of $w$ and $d$. A method called the four-point method extended the solution for larger values of $w$. We then proposed a large scale approximation technique which calculated average 


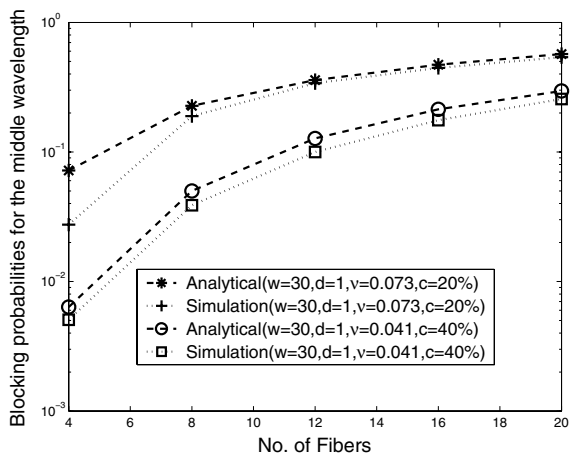

(a) Blocking vs No. of Fibers for $d=1$

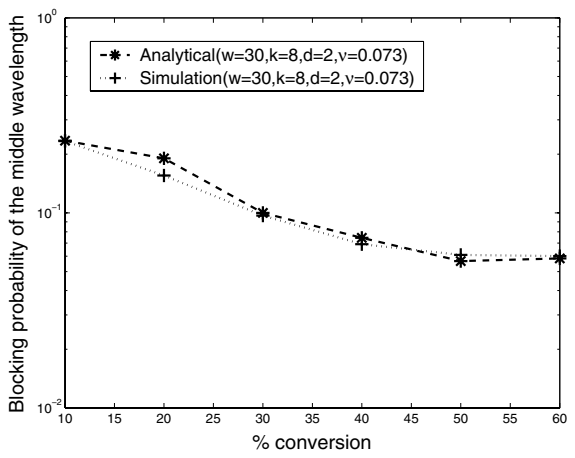

(c) Blocking vs $\%$ conversion for $d=2$

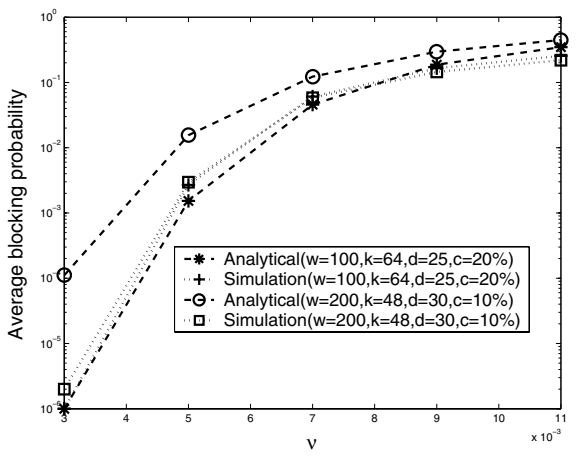

(e) Blocking vs $\nu$ for large systems

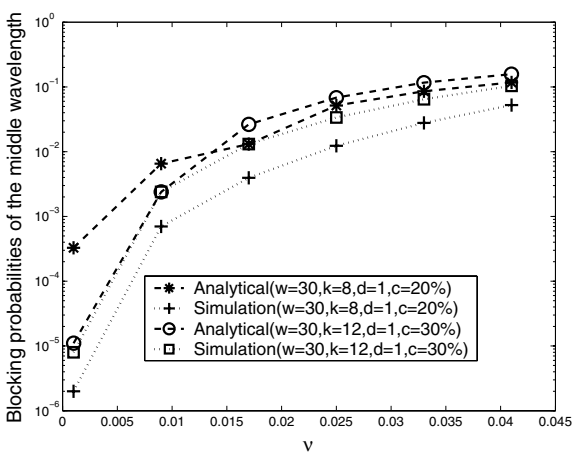

(b) Blocking vs $\nu$ for $d=1$

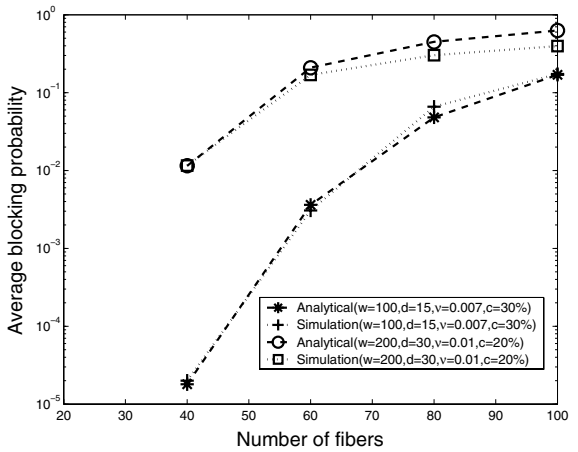

(d) Blocking vs No. of Fibers for large systems

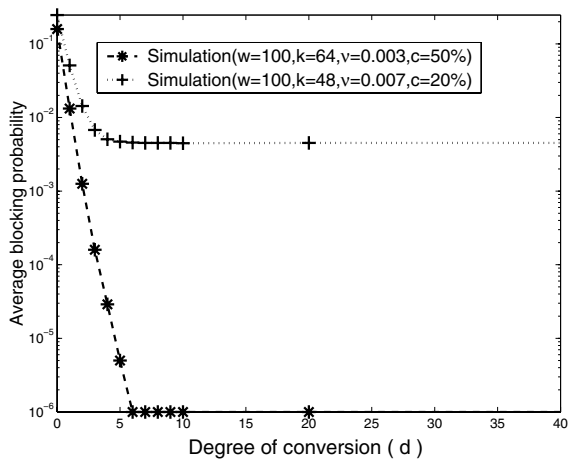

(f) Blocking vs Degree of conversion (d) for large systems 
blocking probabilities for very large values of $w$ and $d$. $d$ has significant impact on blocking when the percentage of converters available is high and/or low arrival rates. When the percentage of conversion is small, the benefits of $d$ saturates very early and large values of $d$ may not be useful.

\section{References}

1. GraphPad Prism. Available for downloading at http://www.graphpad.com, 2004.

2. N. Akar and E. Karasan. Exact calculation of blocking probabilities for bufferless optical burst switched links with partial wavelength conversion. In Proceedings of Broadnets, October 2004.

3. L. Battestilli and H. Perros. An introduction to optical burst switching. IEEE communications magazine, 41:S10-S15, 2003.

4. J. Yates, J. Lacey, D. Everitt and M. Summerfield. Limited-range wavelength translation in all-optical networks. In Proceedings of IEEE INFOCOM, 3:954-961, March 1996.

5. C. Gauger. Optimized combination of converter pools and FDL buffers for contention resolution in optical burst switching. Photonic network communications, 8:139-148, 2004.

6. H. Motulsky and A. Christopoulos. Fitting Models to Biological Data using Linear and Nonlinear Regression: A practical guide to curve fitting. Available for free download from http://www.curvefit.com/, 2003.

7. H. G. Perros. Computer Simulation Techniques: The definitive introduction. Available for free download from http://www.csc.ncsu.edu/faculty/perros/hp.html, 2004.

8. I. Baldine, G. Rouskas, H. Perros and D. Stevenson. Jumpstart: a just-in-time signaling architecture for WDM burst-switched networks. IEEE communications magazine, 40:82-89, 2002.

9. V. Puttasubbappa and H. Perros. An Approximate Queueing Model for LimitedRange Conversion in an OBS Switch. Available at http://www.csc.ncsu.edu/ faculty/perros/Vishwas3.pdf, 2004.

10. X. Qin and Y. Yang. Nonblocking WDM switching networks with full and limited wavelength conversion. IEEE Transactions on Communications, 50:2032-2041, December 2002.

11. J. Ramamirtham and J.Turner. Design of wavelength converting switches for optical burst switching. In Proceedings of IEEE INFOCOM, 1:2032-2041, June 2002.

12. T. Tripathi and K.N. Sivarajan. Computing approximate blocking probabilities in wavelength routed all-optical networks with limited-range wavelength conversion. In Proceedings of IEEE INFOCOM, 1:329-336, March 1999.

13. Y. Xiong, M. Vandenhoute, and H. Cankaya. Control architecture in optical burstswitched WDM networks. IEEE Journal on selected areas in communications, 18:1838-1851, October 2000.

14. Z. Rosberg, H. Vu and M. Zukerman. Performance evaluation of optical burst switching networks with limited wavelength conversion. In Proceedings of ONDM 2003, The 7th IFIP Working Conference on Optical Network Design and Modeling, 2:1155-1169, February 2003.

15. J.Y. Wei and R.I. McFarland. Just-in-time signaling for WDM optical burst switching networks. Journal of Lightwave Technology, 18:2019-2037, December 2000. 